\title{
AUTOMATIC GENERATION OF GEOMETRIC PARAMETERS OF INDIVIDUAL CAULIFLOWER PLANTS FOR RAPID PHENOTYPING USING DRONE IMAGES
}

\author{
G. J. Grenzdörffer ${ }^{1}$ \\ ${ }^{1}$ Chair for Geodesy and Geoinformatics, Universität Rostock, Germany - goerres.grenzdoerffer@ uni-rostock.de
}

Commission I/II, ICWG I/II

KEY WORDS: UAV, Precision Farming, Crop Height, Phenotyping, CHM, Drone

\begin{abstract}
:
Multitemporal drone surveys are a perfect tool to determine various geometric and spectral crop parameters for rapid phenotyping in field trials. Depending on the geometric resolution and the size of the crop, information at leaf level or canopy level can be obtained. The focus of this paper is to demonstrate which geometric properties can be automatically derived from high resolution drone imagery during the vegetation period. For this research approx. 1920 cauliflower with a large genetic variety were planted and monitored by five different drone surveys at an altitude of $20 \mathrm{~m}$, using a high resolution 36 Mpix. RGB-camera. In order to minimize intensive radiometric calibration, BRDF effects and eliminate shade, flights were carried out at overcast skies. After photogrammetric image processing, detailed crop height models (CHM) were computed. 10 distinct crop parameters were derived from a combination of the orthophotos, the CHM and additional information. According to the phenological phase a specific set of parameters was developed for every flight. For instance, the position of the individual plants is computed right after the first flight. For the flight prior to harvesting, an algorithm for the head diameter and the curvature of the cauliflower heads was developed. Geometric parameters are generally better suited for automation, because they require less specific ground truth or reference information, than spectrally derived biophysical parameters.
\end{abstract}

\section{INTRODUCTION}

Unmanned aerial systems (UAS) are in many ways a a very convenient tool for precision farming applications in general and field trials in particular, as many plant-relevant information can be collected at defined times, quickly, without contact, objectively and automatically, e.g. (Hunt \& Daughtry, 2018), (Maes \& Steppe , 2018). The topic of phenotyping is also of great interest in the context, since, depending on the culture, an identification of the individual plant is possible, and the growth process can be individually recorded, compared and evaluated (Madec et al. 2017). Most of the published drone related phenotyping research is related to common crops such as wheat, barley, corn, sorghum etc. Vegetables, regional and less common crops are rarely in the focus.

Plant phenotyping refers to a quantitative description of the plant's anatomical, ontogenetical, physiological and biochemical properties (Walter et al., 2015). The continuous monitoring of the crop growth with drones providing a large set of different parameters opens up many new applications, e.g. to record and document the course of plant development and thus also the underlying processes such as fertilizer intake and the course of plant diseases. Generally speaking geometric, spectral and temporal properties may derived from the plants, figure 1 . However, for efficient phenotyping this assumes that the data processing of the drone data and data analysis for the evaluation by the agronomist is more or less automatic.

The term "resolution" is very central to remote sensing because it determines what can be extracted from the aerial imagery. A distinction is made between spatial, radiometric, spectral and temporal resolution. When using drones, there is a nearly free choice of spatial resolution. this multiplies the application possibilities, because the free scalability allows both the selective view of the individual plant and the large overview of the plant canopy from a bird's eye view.

In practice, there are two ways to vary the ground resolution, often also referred as ground sampling distance (GSD). On the one hand, about the altitude, i.e. flying at higher altitudes, the ground resolution becomes lower. On the other hand by changing the focal length, thus at the same altitude a higher GSD can be achieved with a longer focal length (zoom). Closely related to GSD is the spatial scale or observation level, which extends from the leaf level via the individual plant level to the plot and the canopy level. Ground resolution is of great importance in many respects and has various consequences:

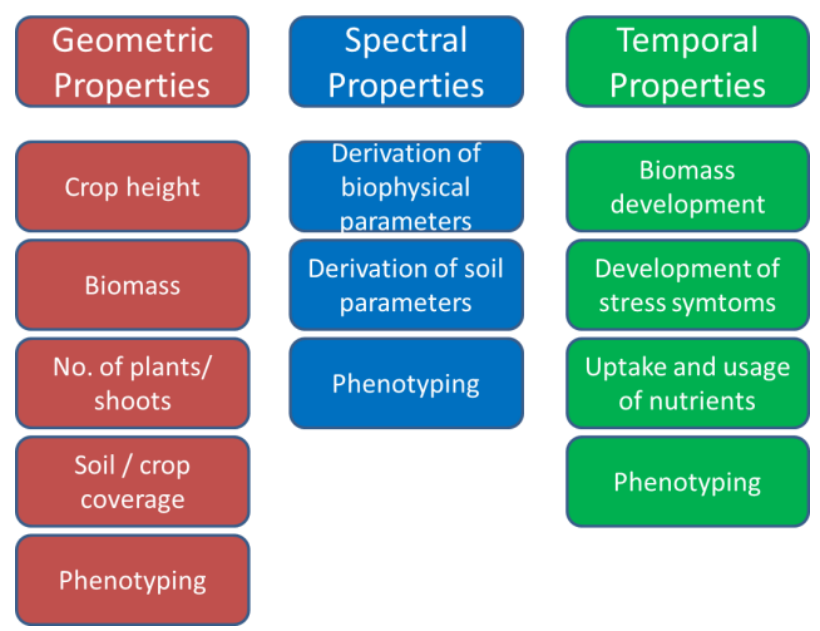

Figure 1: Overview of various information or plant properties for field trials, which can be derived from UAS images 
1. The GSD determines the recognizability or separability of individual objects and thus also the scale level. At the highest GSD in the mm range, individual leaves or their structure can be identified. These high resolutions are only generated in the laboratory with high resolution cameras or microscopes close to the plants, e.g. (Walter et al., 2015). At ground resolutions of $0.5-4 \mathrm{~cm}$, larger leaves and single plants can be clearly separated, depending on the plant species and phenological stage. At GSD of $>4 \mathrm{~cm}$, the transition to the canopy level begins. Individual plants can no longer be separated and the sensor records a mixed signal that consists of several components (plant, shadow, soil), as we know from aircraft or satellite remote sensing.

2. The flying and storage efforts increase exponentially with the ground resolution. Halving the ground resolution means at the same time quadrupling the amount of data and the flight time.

3. Various problems are associated with the scale transitions. This applies in particular to the fact that the spectral reflection properties change greatly depending on whether they are measured on a single leaf (leaf level) or represent an average value of several components, such as soil, plant and shade (canopy level). Since the sun acts as a light source, the irradiation conditions change depending on the position of the sun, the light intensity (cloud cover, season) and the plant architecture. In addition, the reflection properties change continuously during plant growth and due to stress. To short cut it here, a quantitative derivation of biophysical parameters in a scale and across scale boundaries is very complex and the subject of many research activities. To sum up, it will take be some time before robust and fully automated methods are available.

The special and unique advantage of UAS-supported remote sensing is the high temporal resolution with which the highresolution remote sensing data can be collected. One can flexibly react to changing weather conditions on site and - if possible take full advantage of the sunny or fully cloud covered minutes or hours. However, this requires that rapidly changing radiation changes are detected at the same time. Suitable multispectral sensors such as the Sequoia sensor from Parrot or MicaSense are now available on the market. For common RGB-cameras such radiometric calibration devices do not exist.

Table 1: Comparison between the use of a radiometrically calibrated camera vs. a drone flight with overcast sky

\begin{tabular}{|c|c|c|}
\hline Method & Advantages & Disadvantages \\
\hline \multirow{3}{*}{$\begin{array}{l}\text { Parallel } \\
\text { measurement } \\
\text { of incoming } \\
\text { and reflected } \\
\text { sunlight }\end{array}$} & $\begin{array}{l}\text { Flight under sunny and } \\
\text { cloudy conditions (more } \\
\text { flight days) }\end{array}$ & \multirow{2}{*}{$\begin{array}{l}\text { The multispectral camera } \\
\text { system does not solve the } \\
\text { BRDF-problem at the leaf } \\
\text { and (canopy) level }\end{array}$} \\
\hline & $\begin{array}{l}\text { Direct measurement of } \\
\text { reflectance }\end{array}$ & \\
\hline & $\begin{array}{l}\text { Temporal and } \\
\text { multisensoral } \\
\text { comparisons possible }\end{array}$ & Costly special sensor \\
\hline \multirow[t]{3}{*}{$\begin{array}{l}\text { Flight with } \\
\text { overcast sky }\end{array}$} & Very simple solution & $\begin{array}{l}\text { Maybe insufficient light } \\
\text { available }\end{array}$ \\
\hline & $\begin{array}{l}\text { No additional hardware } \\
\text { necessary }\end{array}$ & Lower number of flight days \\
\hline & No shades & No absolute reflectance \\
\hline
\end{tabular}

Flying below the clouds can greatly simplify spectral calibration, since the bi-directional reflection properties (BRDF) of the direct sunlight do not have to be taken into account and modelled.
Flights at overcast skies are generally better suited for further (automatic) image analysis see table 1 for the pros and cons. On the basis of a systematic drone survey, corresponding control points or RTK-GNSS technology on board the UAS and a solid photogrammetric data processing, UAS images provide not only two-dimensional images, but three-dimensional geodata that can be evaluated and interpreted accordingly (automatically). In summary, a lot of information can be derived from the UAS images, where the geometric, spectral or temporal properties of the plant development are in the foreground. Geometric parameters, such as the height of growth or the degree of soil cover, can be calculated automatically as far as possible with inexpensive sensors (color digital camera) and will therefore be presented in more detail in the following.

Depending on the selected ground resolution (canopy level or leaf level) and the cultivar, geometric parameters, such as the height of the plant (e.g. Grenzdörffer 2014), or the degree of plant or soil cover e.g. Torres-Sanchez et al., 2014, can be calculated nearly automatically with common digital imaging sensors (color digital camera). While the focus of this paper is on the derivation of geometric parameters, it is of course also possible to use the spectral values of an RGB camera to correlate them, for example, with biophysical parameters, e.g. (Rasmussen et al. 2016) or (Schirrmann et al. 2016).

The aim of the presented cauliflower experiment in the summer of 2016, was to detect the individual plants as automatically as possible after planting, to observe the growth of the plants and to automatically determine the size and shape of the cauliflower heads. The trial consisted of a comparison of 240 different double haploid lines of cauliflower ( 8 plants / line, i.e. about 1,920 plants). Due to the specific genetic properties of the different lines, the cauliflower plants develop quite differently in terms of the duration of the vegetation period, the overall appearance, the number and form of leaves, the size and form of the cauliflower head etc.

\section{DATA}

In order to identify, analyze and evaluate individual plants, five low altitude UAS surveys (flight height $20 \mathrm{~m}$ ) were carried out. The total size of this experiment was approximately $23 \times 23 \mathrm{~m}$ or $530 \mathrm{~m}^{2}$. The surveys were always carried out at overcast sky conditions, to minimize BRDF effects and shades.

A falcon 8 drone from Intel, equipped with a Sony $7 \mathrm{R}$ camera with a 36 MegaPixel RGB-Sensor was used for the image surveys. Per flight approximately 100 images were acquired. The ground resolution resulting from the altitude, the sensor size and the focal length was approximately $3 \mathrm{~mm}$ per pixel. The surveys were carried out systematically with a longitudinal and transverse overlap of $80 \%$ and $60 \%$, respectively.

\subsection{UAS Surveys}

The first flight on 05-10-2016 (DOY = 135) was carried out right after the crops were planted into the field. At this phenological stadium the plants have between 3 - 5 leaves. Most of the plants are still laying on the ground, whereas other plants are already erected.

Before the second flight on 06-14-2016 (DOY = 166) the net against specific insects was lifted. Due to differences in irrigation, the crop development was quite variable. 
At the time of the third flight on 06-22-2016 (DOY = 174) the first cauliflower heads of $1-2 \mathrm{~cm}$ in diameter were observed manually, but they are not visible in the images due to cover leaves. The vast majority of the plants were still in the vegetative phase.

12 days later, at the fourth drone flight on 07-04-2016 (DOY = 186) cauliflower heads were observed manually in 174 of 232 lines. In the orthoimage of that epoch 338 out of 1913 heads are visible. The average size of the cauliflower head was $4.8 \mathrm{~cm}$, figure 2 .

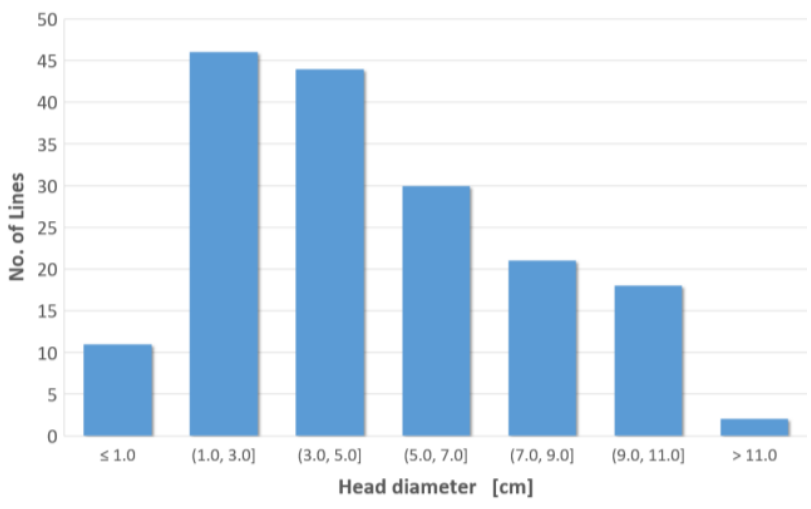

Figure 2: Diameter of cauliflower heads measured at 07-04-2016, $\mathrm{N}=174$ Lines

Prior to final harvesting, 10 days later, at the fifth flight on 07 14-2016 (DOY = 196) 281 plants were already harvested and 1.632 plants remained on the field. In total, cauliflower heads could be observed in 222 of 240 lines. Thus, 18 lines (genotypes) did not produce any cauliflower heads. The average size of the cauliflower head was $11.8 \mathrm{~cm}$, figure 3 . This is significantly smaller than most commercially available products.

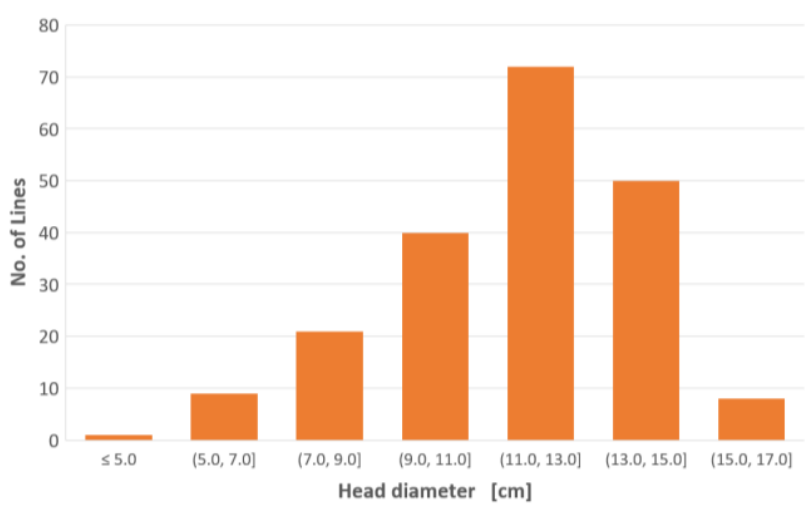

Figure 3: Diameter of cauliflower heads measured at 07-16-2016, $\mathrm{N}=222$ Lines

\subsection{Photogrammetric Data Analysis}

The images were photogrammetrically processed with the software Photoscan Professional (Agisoft LLC) and 6 signalized control points. The absolute position and height accuracy of 1 - 2 $\mathrm{cm}$ is limited by the accuracy of the control points, which were measured with RTK-GNSS. Nevertheless, a basic requirement for multitemporal studies is fulfilled, since the different epochs match geometrically. The results of the photogrammetric processing were the $3 \mathrm{D}$ point cloud, the derived digital surface model (DOM), and a digital orthophoto with a GSD of $3 \mathrm{~mm}$. A digital terrain model (DTM) is derived from the first flight. The subsequent digital crop models (CHM) are generated by subtracting the digital surface models of the epochs $2-5$ from the DTM of the first flight.

During the photogrammetric processing the 3D point cloud and DOM is often calculated at a lower resolution than the original GSD of the images for various reasons and the outcome is often also filtered or smoothed to delete outliers or other unwanted 3D points. The main reason for using a reduced resolution are the associated time savings, since stereo or multi-ray matching is a computationally very intensive step. The time required and the number of calculated points increases exponentially, while the absolute height accuracy barely increases, or rather the noise increases. The following comparison on the basis of 104 images shall clarify this.

Table 1: Comparison of derived 3D point clouds depending on the software settings of Agisoft Photoscan Professional (v 1.25) and the SURE software

\begin{tabular}{llllll}
\hline & Lowest & Low & Medium & High & SURE \\
\hline $\begin{array}{l}\text { Duration* } \\
\text { (Min.) }\end{array}$ & ca. 2 & ca. 20 & ca. 110 & ca. 583 & ca. 700 \\
\hline $\begin{array}{l}\text { Points } \\
\text { (Mio.) }\end{array}$ & 0.69 & 3.03 & 12.96 & 53.34 & $2,524.9$ \\
\hline GSD DEM & $5 \mathrm{~cm}$ & $2.5 \mathrm{~cm}$ & $1.25 \mathrm{~cm}$ & $0.625 \mathrm{~cm}$ & $0.3 \mathrm{~cm}$ \\
\hline
\end{tabular}

* Computing time on PC with 32 GB RAM

According to the resolution topic in the introduction, the processing level of the 3D point cloud decides whether the 3D data is suited for canopy level related parameters or at a higher level of detail for leaf level parameters. For instance at the highest resolution the 3D-point cloud delivers 3D-details about the single leaves and the shape of the cauliflower head, highlighted in Figure 8.

\section{RESULTS}

During the vegetation period, the following geometric parameters of the cauliflower plants were determined:

- Exact location of the plants / parcels (1st flight)

- Available standing space per plant (1st flight)

- Degree of plant coverage (2nd - 4th flight)

- Cauliflower head diameter (4th and 5th flight)

- Height of the cauliflower head (5th flight)

- Curvature of the cauliflower head (5th flight)

From the single plant information, several crop and parcel specific parameters were computed:

- Assignment of individual plant to parcel number

- Homogeneity of plants within a parcel

- Crop growth (height increase) between the different flights

- Change in the degree of crop coverage per parcel

In the following, the above-mentioned parameters will be described in more detail. For automation, the subsequently described procedures for the derived geometric parameters are implemented as ArcGIS models. 


\subsubsection{Exact location of the plants / parcels (1st flight)}

The exact location of the plants is the foundation for further single plant related data analysis. In a first step the green plants are separated from the soil background. For this purpose, the vegetation index VARI is used (Stark et al., 2000). Above a threshold, a reliable separation between soil and plants could be achieved. Subsequently, the identified vegetation pixels are morphologically filtered, vectorized, buffered and seedlings are separated from other plants or misallocations by a size filter and the parcel boundaries. The classification results are nearly $100 \%$. (1908 of 1913 plants were detected automatically).

Afterwards the individual plants are associated to the parcels, thus every plant gets parcel specific attributes, such as the genotype etc. As most of the seeded plants are still lying on the ground, the automatically determined position thru image analysis is not necessarily the location of the stem of the plant. The position of the center of final cauliflower head will also not be at the exact position of the initial plant, but within a range of $\pm 10 \mathrm{~cm}$, because the main axis of the plant is not always straight. This may cause problems to spatially join the cauliflower head information to the single plant. Figure 4 shows the different locations of the center of the automatically computed plant position, the location of the stem of the plant and the diameter and center of the final cauliflower head.

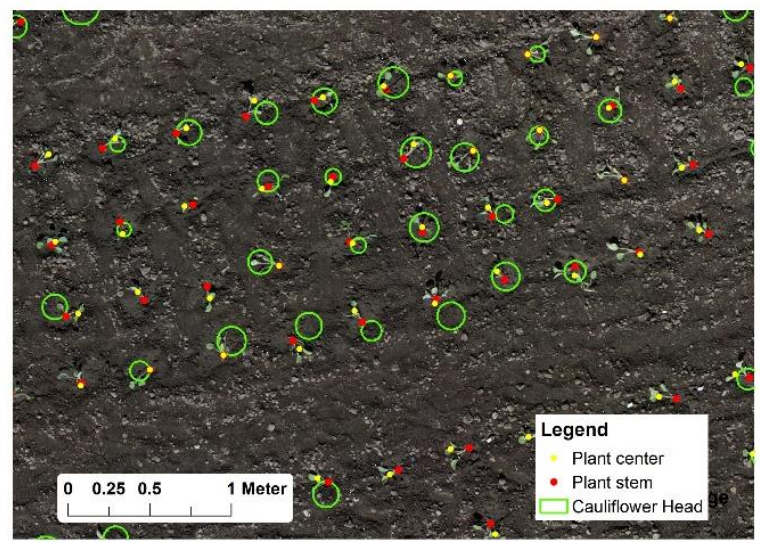

Figure 4: Comparison of location of the automatically computed plant position vs. the stem of the plant and the diameter of the final cauliflower head

\subsubsection{Available standing space per plant (1st flight)}

The predefined plant to plant spacing was set to $45 \mathrm{~cm}$. However, the plants were planted by hand and the prior defined spacing may differ from plot to plot. Furthermore, plants at the edge of the parcels as well as plants adjacent to fails have more individual space for further growth. The determination of the individual space is computed thru a Thiessen polygon analysis, Figure 5.

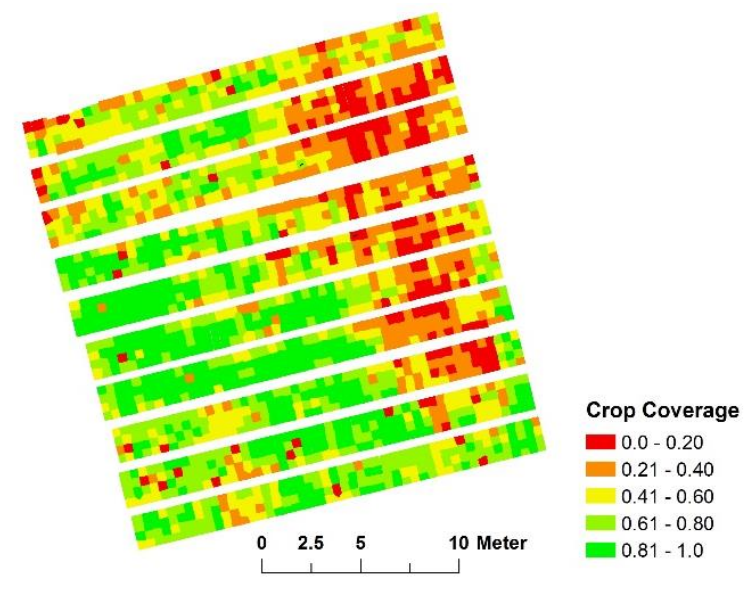

Figure 5: Proportion of crop coverage per single plant of the 2nd drone flight. Red plants are either small or not growing well, whereas the green plants cover most of the available space

3.1.3 Degree of plant coverage and plant volume (2nd - 4th flight)

The percentage of the vegetation coverage is calculated with the help of the CHM. All pixels higher than $3 \mathrm{~cm}$ are considered to be vegetation and all other pixels below this threshold are bare ground pixels. Additionally, the mean height of the vegetation covered pixels per plant and per parcel is determined for the flights $2-4$. The values are associated to the individual plants and the parcels, see figures 5 and 6 .

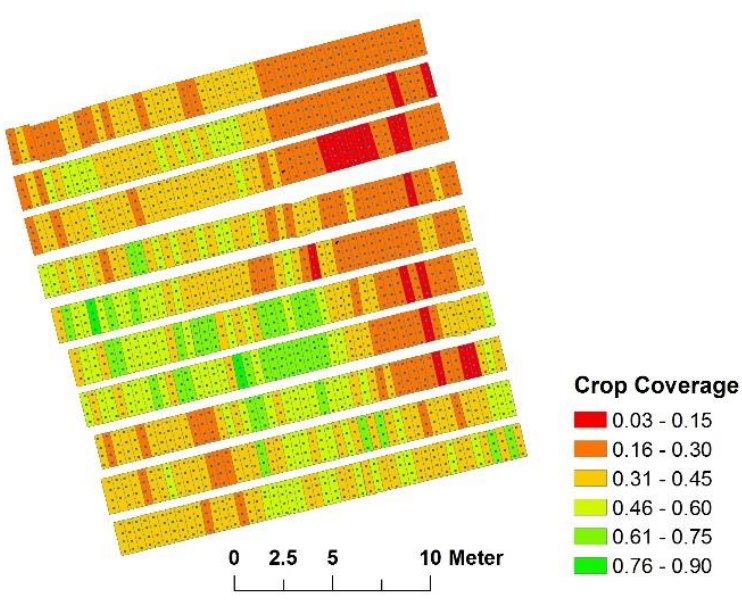

Figure 6: Proportion of crop coverage per parcel of the 2nd drone flight. The location of the individual plants (black dots) were computed in the $1^{\text {st }}$ flight.

\subsubsection{Cauliflower head diameter (4th and 5th flight)}

With the white color, cauliflower heads are spectrally unique, compared to the rest of the ortho image. This offers an opportunity to automatically determine the size of the cauliflower heads. The procedure is composed of five different steps:

1. Segmentation and multispectral classification (SVM) in order to separate the white cauliflower heads from the rest of the image content. The cauliflower heads shall be single segments. After the classification of the segments, the white ones are selected for further processing. 
2. Raster to vector conversion of the cauliflower heads candidates.

3. Filtering of erroneous candidates such as white or brown leaves of already harvested plants due to their size, height, location and form. The minimum size was set to $0.1 \mathrm{dm}^{2}$ and the maximum size $4.5 \mathrm{dm}^{2}$. The ratio between the perimeter and area was set to a limit of 2.2 and the minimum altitude of the head was set to $20 \mathrm{~cm}$ above the ground.

4. Smoothing of the shape of the candidates and calculation of the bounding circle.

5. Calculation of the head diameter as an additional attribute to the plants thru a spatial join, figure 7 .

\subsubsection{Height of the cauliflower head (5th flight)}

The genetics of the different varieties determine the point in time from the vegetative to the generative phase of the plant. Cauliflower plants with a lower head height above ground are generally bigger at the time of harvest, compared to plants that initiate the generative phase at a later time. Therefore, it is of interest to determine the average height of the cauliflower head as an additional attribute for every plant, Figure 7.

\subsubsection{Curvature of the cauliflower head (5th flight)}

Depending on the genetics of the specific variety the curvature of the cauliflower head may differ from flat to strongly curved. The curvature of the head is a quality measure for the producer and the market. In accordance with the high image resolution, the curvature of the cauliflower heads may be determined automatically.

1. Computation of the center point of the previously detected cauliflower head.

2. Calculation of the height at the center point of the cauliflower head from the crop height model

3. Calculation of the ratio between the mean height and the height in center. Crops with ratio of $<1$ are considered to have a flat head. Crops with a ratio between 1 and 1.04 are classified as slightly curved and cauliflower heads with a ratio of $>1.04$ are strongly curved.

The following example shows this for cauliflower plants, in which the head diameter, the curvature of the head and the stature height was automatically detected.

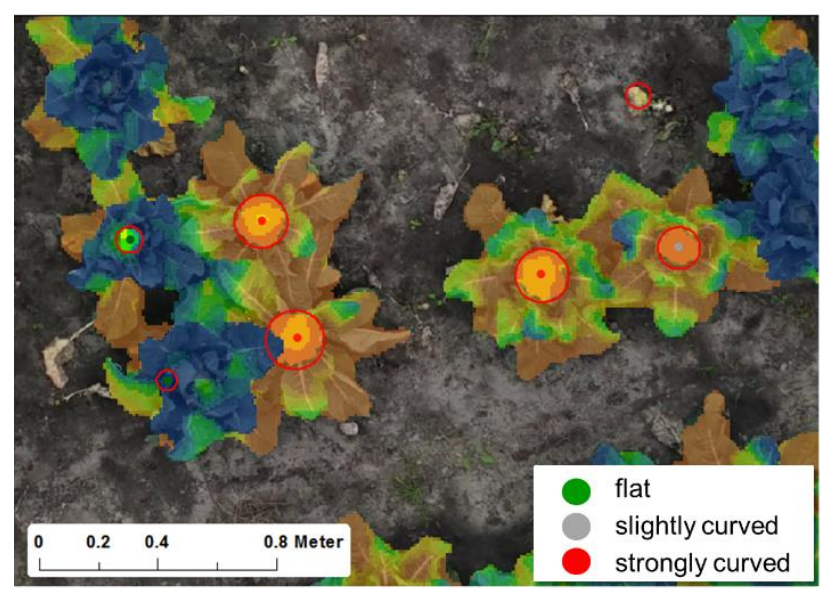

Figure 7: Example of cauliflower plants with automatically detected head diameter, curvature of the head and the stature height

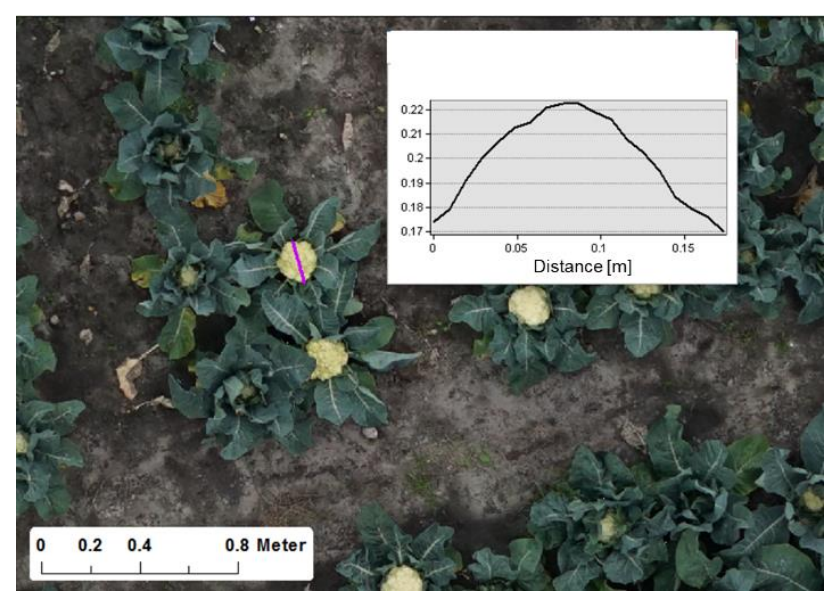

Figure 8: Example of a strongly curved cauliflower head, determined from the detailed crop height model

\subsection{Accuracy assessment}

Do the image based automatic and manual digitizing of the diameter of the cauliflower heads deliver the same results as the common manual approach? This was the most important question. The answer is - generally spoken - yes, but a comparison is not so easy, because the manual reference measurements with a ruler were not taken at the same day, but two days later. Additionally the manual reference measurements were rounded to full $\mathrm{cm}$ values and only averages for single lines (2 repetitions with max. 8 plants) are available. For the comparison only lines with 7 or 8 plants were selected and the variation between the plants shall be small (low standard deviation). At first the manually digitized diameters from the ortho images and the automatically derived head diameters are compared, Figure 9.

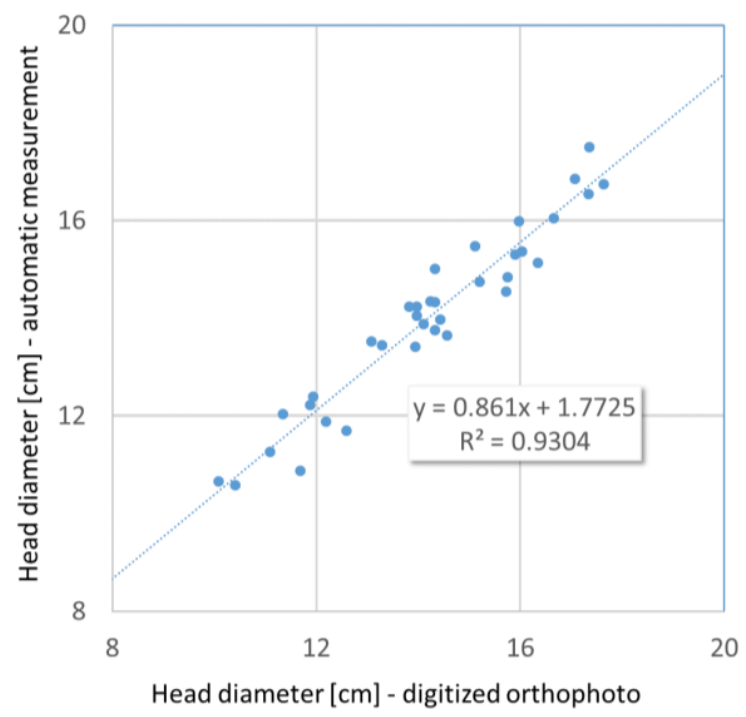

Figure 9: Regression between the automatically measured and digitized head diameters $(\mathrm{N}=35$ lines $(245$ individual measurements)) 


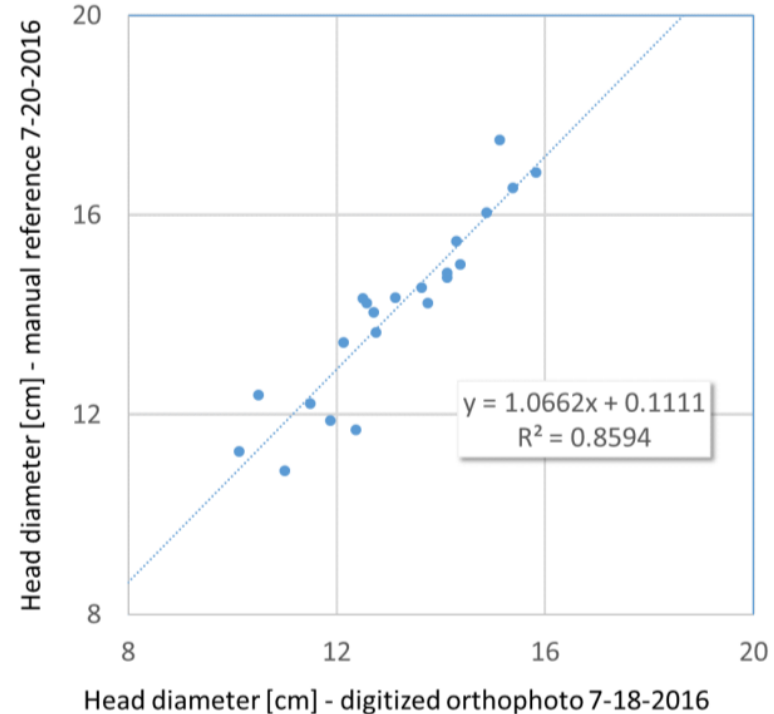

Figure 10: Relationship of cauliflower head size measured manually vs. digitized orthophoto, $\mathrm{N}=25$ lines $(200$ measurements)

The difference in the average diameter between the automatically derived head diameters and the digitized values from the orthophotos is only $0.2 \mathrm{~cm}$, with a standard deviation of $0.55 \mathrm{~cm}$. The automatic measurements often failed with very small cauliflower heads, therefore the small ones were not considered in the comparison, shown in Figure 9.

The next comparison is between the manual measurements in the field taken at 2 days later vs. the digitized values from the orthophotos, Figure 10.

The last comparison is between the manual reference and the automatically derived cauliflower head diameters, Figure 11.

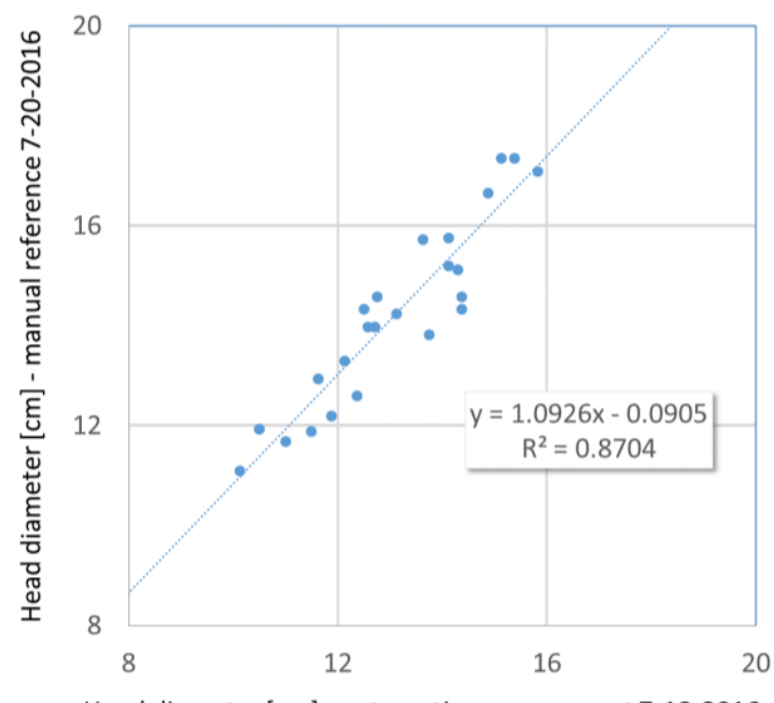

Figure 11: Relationship of cauliflower head size manually measured vs. automatically measured, $\mathrm{N}=22$ lines (184 measurements)
Within 2 days from the automatic measurement to the manual reference measurements, the average head diameter has grown $1.2 \mathrm{~cm}$.

The manual measurement of the cauliflower head is more reliable for small diameters and overlying bracts and provides most counts $(1,468)$. Manual digitization of the cauliflower head diameters in the orthophoto delivers comparable values to the manual measurements as well as to the automatic approach, but fewer heads $(1,146)$ could be counted due to overlying bracts, which made precise measurements in the orthophotos impossible. Automatic procedures deliver similar results, but recognizes even fewer heads (966), than the other two approaches, because for automatic detection the heads have to have certain minimum size. Overall, there is a very good correlation between the manual reference measurements and the automatic measurements.

\section{CONCLUSIONS}

With high resolution drone imagery, a continuous coverage of the crop growth of cauliflower on the level of individual plants is now possible. It should be noted that with the determination and characterisation of the individual plants, additional data for further statistical analysis is readily available, such as information on the variability of the plants within a parcel. In addition, individual outliers, edge effects, etc. can be identified.

A large number of geometric plant phenotyping parameters had to be specifically developed for cauliflower plants, as there is no other case described in the literature. Not all of the derived parameters are of direct use for the agronomists or the plant breeders, because they are currently not compatible with other more commonly used parameters. Future research is necessary to validate the results.

Beside the automatically derived information, the very high resolution UAS image data provides much more information that is not or little reflected in the standard statistical approach on the parcel level. For instance, leaf position and leaf shape, pitting by insects, weed infection, damage from bird eating, the degree of coverage and much more.

\section{REFERENCES}

Grenzdörffer G., 2014. Crop Height Determination with UAS Point Clouds.- Int. Arch. Photogramm. Remote Sens. Spatial Inf. Sci., XL-1, pp. 135-140, doi:10.5194/isprsarchives-XL-1-1352014.

Hunt, R.; Daughtry, C., 2018. What good are unmanned aircraft systems for agricultural remote sensing and precision agriculture? Int. Journal of Remote Sensing 39 (15-16), p. 53455376. DOI: $10.1080 / 01431161.2017 .1410300$.

Madec, S. Baret, F., Solan, F. Benoît de; Thomas, S.; Dutartre, D.; Jezequel, S. et al., 2017. High-Throughput Phenotyping of Plant Height: Comparing Unmanned Aerial Vehicles and Ground LiDAR Estimates. Frontiers in plant science 8, p. 2002. DOI:10.3389/fpls.2017.02002.

Maes, W., Steppe, K., 2018. Perspectives for Remote Sensing with Unmanned Aerial Vehicles in Precision Agriculture. In: Trends in plant science. DOI: 10.1016/j.tplants.2018.11.007

Rasmussen J., Ntakos G., Nielsen J., Svensgaard J., Poulsen R.N., Christensen S., 2016. Are vegetation indices derived from 
consumer-grade cameras mounted on UAVs sufficiently reliable for assessing experimental plots? Eur. J. Agron. 2016, 74, pp. 7592.

Schirrmann M., Giebel A., Gleiniger F., Pflanz M., Lentschke J., Dammer K.-H., 2016. Monitoring Agronomic Parameters of Winter Wheat Crops with Low-Cost UAV Imagery.- Remote Sens. 2016, 8, 706; doi:10.3390/rs8090706.

Stark R., Gitelson A., Grits U., Rundquist D., Kaufman Y., 2000. New technique for remote estimation of vegetation fraction: principles, algorithms and validation, Aspects of Applied Biology, 60, pp. 241 - 246.

Torres-Sánchez J. Peña J.M., De Castro A.I., López-Granados F., 2014. Multi-temporal mapping of the vegetation fraction in earlyseason wheat fields using images from UAV.- Computers and Electronics in Agriculture 103, pp. 104-113.

Walter, A., Liebisch, F., \& Hund, A., 2015. Plant phenotyping: from bean weighing to image analysis. Plant methods, 11, 14. doi:10.1186/s13007-015-0056-8 